\title{
Edge-Clique Graphs and the $\lambda$-Coloring Problem
}

\author{
Tiziana Calamoneri \& Rossella Petreschi \\ Dept. of Computer Science, University of Rome "La Sapienza" - Italy \\ via Salaria 113, 00198 Roma, Italy. \\ e-mail address:\{ calamo, petreschi $\} @$ dsi.uniroma1.it
}

\begin{abstract}
This paper deals with edge-clique graphs and with the $\lambda$-coloring problem when restricted to this class.

A characterization of edge-clique graphs of outerplanar graphs is given; a complete description of edge-clique graphs of threshold graphs is presented and a linear time algorithm for $\lambda$-coloring the edgeclique graph of a threshold graph is provided.

A survey on the $\lambda$-coloring problem, when restricted to edge-clique graphs, is reported.

keywords: edge-clique graphs, $\lambda$-coloring, outerplanar graphs, threshold graphs.
\end{abstract}

\section{Introduction}

The edge-clique graph $K_{e}(G)$ of a graph $G$ is a graph whose vertices correspond to the edges of $G$ and two vertices are adjacent whenever the corresponding edges of $G$ belong to a common clique.

Edge-clique graphs appeared implicitly in literature in 1978 in a paper by Kou, Stockmeyer and Wong concerning keyword conflicts [19], but the first systematic paper dealing with them is due to Albertson and Collins and was published in 1984 [1].

In [1] edge-clique graphs are inspired by the graph representing the incidence system arisen by the following formulation of the four color theorem: the edges of a triangulation of the plane can be 3-colored such that the edges incident with every triangle are colored distinctly.

The fact that edge-clique graphs were born by a problem, perhaps the most famous, in the field of graph coloring [18], suggested us to explore, restricted to edge-clique graphs, a very actual coloring problem related to the design of wireless communication systems: the $\lambda$-coloring problem.

The $\lambda$-coloring problem consists in an assignment of colors from the integer set $(0 . . \lambda)$ to the vertices of a graph $G$ such that vertices at distance at most two get different colors and adjacent vertices get colors which are at least two apart. The aim is to minimize $\lambda$.

This problem was born in the context of mobile computing (see, for instance $[12,14,16]$ ). The task is to assign radio frequencies to transmitters at different locations without causing interference. This situation is modeled by a graph, whose vertices are the radio transmitters/receivers, and the adjacencies indicate possible communications and, hence, interference. The aim is to minimize the frequency bandwidth, i.e. $\lambda$.

In the general case, the $\lambda$-coloring problem is NPcomplete, as the most common problem of the vertex coloring problem is. Therefore it is interesting to investigate special classes of graphs for which it is possible to find either tight bounds for the number of necessary colors or approximating solutions.

This paper deals with the $\lambda$-coloring problem restricted to edge-clique graphs. Namely, a linear time algorithm for $\lambda$-coloring the edge-clique graph of a threshold graph is provided. Moreover, we characterize edge-clique graphs of outerplanar graphs as particular outerplanar graphs, so the same results concerning the $\lambda$-coloring problem of outerplanar graphs [7] hold for their edge-clique graphs.

The paper is organized as follows.

In the next section terminology and definitions useful in the rest of the paper are recalled.

Edge-clique graphs are closely examined in $\mathrm{Sec}-$ tion 3 and their main properties are pointed out.

Section 4 characterizes edge-clique graphs of outerplanar graphs and shows that edge-clique graphs of outerplanar graphs are particular triangulated outerplanar graphs.

Section 5 surveys most of the known results concerning $\lambda$-coloring problem and discusses those concerning edge-clique graphs of different classes of graphs.

Finally, in Section 6, an algorithm for $\lambda$-coloring the edge-clique graph of a threshold graph is presented and the proofs of its correctness and linearity are provided. 


\section{Background}

In all this paper we consider only finite, simple, loopless graphs $G=(V, E)$, where $V$ is the vertex set and $E$ is the edge set of $G$; for references see the book [5], when another one is not explicitly cited.

A vertex $x \in V$ is called universal (isolated) if it is adjacent to all other vertices of $V$ (no vertex in $V$ ); if $x$ is an universal (isolated) vertex, then its degree is $\operatorname{deg}(x)=|V|-1(\operatorname{deg}(x)=0)$.

A graph $G=\left(\left\{v_{1}, v_{2}, \ldots v_{k}\right\}, \emptyset\right)$ is a null graph if its edge set is empty, independently by the dimension of the vertex set, i.e. if all its vertices are isolated.

A graph $G$ is planar if it can be drawn in the plane without crossings. Such a drawing is called embedding. A graph is outerplanar if it can be embedded in the plane so that every vertex lies on the boundary of the outer face.

A graph $I=\left(V_{I}, E_{I}\right)$, where $V_{I} \subseteq V$ and $E_{I}=$ $E \cap\left(V_{I} \times V_{I}\right)$ is said to be induced by $V_{I}$. A graph $I$ induced by the subset $V_{I} \subseteq V$ is called:

- complete or clique if any two distinct vertices in $V_{I}$ are adjacent in $G$;

- stable or independent if no two vertices in $V_{I}$ are adjacent in $G$.

We call $q(G)$ the number of maximal cliques in a graph $G$.

A graph $G$ is said to be split if there is a partition $V=V_{K} \cup V_{S}$ of its vertices such that the induced subgraphs $K$ and $S$ are complete and stable, respectively.

For any graph $G$, let $N(x)$ be the set of $x$ 's adjacent vertices. Then, the vicinal preorder $\preceq$ on $V$ is defined as follows: $x \preceq y$ iff $N(x)-y \subseteq N(y)-x$.

A graph $G=(V, E)$ is a threshold graph if and only if it is a split graph and all its vertices are in vicinal preorder (see Fig. 9.a).

Let $F$ be a family of subsets of some sets. The intersection graph of $F$ is a graph whose vertices are associated to the subsets of $F$, two vertices being adjacent if the corresponding pair of subsets intersect.

A graph $K(G)$ is said the clique graph of the graph $G$, if it is the intersection graph of the maximal cliques of $G$ (see Fig. 1), [4, 22, 23].

A graph $K_{e}(G)$ is said the edge-clique graph of the graph $G$, if its vertices are the edges of $G$ and two vertices in $K_{e}(G)$ are joined by an edge if the corresponding edges in $G$ are contained in the same clique (see Fig. 2), [1].
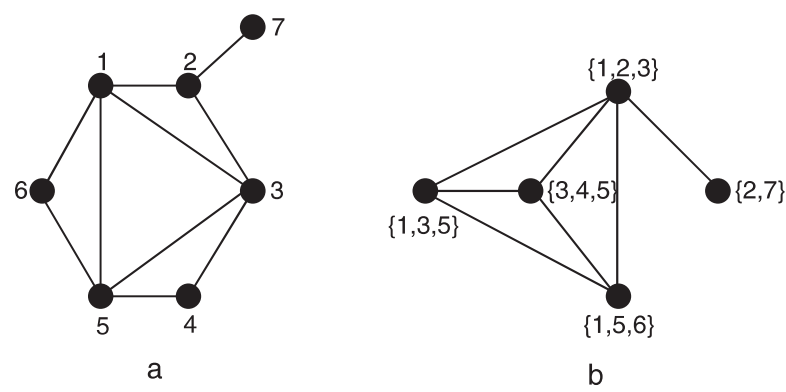

Figure 1: a. A graph $G$; b. its clique graph $K(G)$.

From 1984 to nowadays much work has been done on edge-clique graphs; a not exhaustive list of papers is $[9,10,11,13,21,25,26]$. In the next section we summarize some results on edge-clique graphs useful for the rest of the paper.

\section{Edge-clique graphs}

From the definition of edge-clique graph the following two facts hold:

Fact 3.1 If $G$ is a clique, its edge-clique graph $K_{e}(G)$ is a clique, too.

Fact 3.2 If $G$ is either a path, or a bipartite graph, or an hexagonal or squared tiling, or a cycle with at least 4 vertices, then its edge-clique graph $K_{e}(G)$ is $a$ null graph.

Notice that, in Fact 3.2, cycles with 3 vertices are omitted because they are cliques. Furthermore, they are the only fixed point with respect to $K_{e}$ transformation.

Theorem 3.3 [25] The edge-clique graph $K_{e}(G)$ of a chordal (strongly chordal) graph $G$ is still chordal (strongly chordal).

Observe that non-isomorphic graphs may have isomorphic edge-clique graphs, as shown in Fig. 2 [9].

Theorem 3.4 [1] If $G$ is a planar graph and it contains cliques of dimension not greater than 3 , then its edge-clique graph $K_{e}(G)$ is planar.

The hypothesis that cliques with more than 3 vertices do not exist is substantial for Theorem 3.4. Indeed, if cliques of dimension 4 are present in $G$, not only $K_{e}(G)$ is not planar anymore, but it may also be very complicated, as shown in Fig. 3.a and 3.b. Cliques of higher dimension cannot be in $G$ since are not planar. Finally we have to underline that the converse of Theorem 3.4 does not hold, see Petersen graph (Fig. 3.c) as an example. 


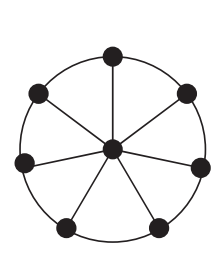

a

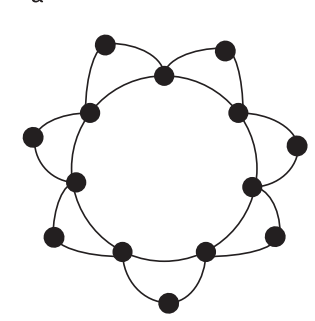

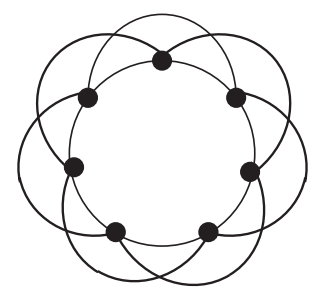

b
Figure 2: Edge-clique graphs of non isomorphic graphs can be isomorphic: a. $G_{1}$; b. $G_{2} ;$ c. $K_{e}\left(G_{1}\right)=K_{e}\left(G_{2}\right)$.

Fact 3.5 [1] If in $G$ there is a clique of dimension $k$ in its edge-clique graph $K_{e}(G)$ there is a clique of dimension $\left(\begin{array}{l}k \\ 2\end{array}\right)$. Viceversa holds.

From the previous fact, it follows that cliques in an edge-clique graph can have only dimension equal to a binomial coefficient, then cliques of dimension $2,4,5,7,8,9, \ldots$ cannot be in. Consequently, since $K_{e}(G)$ cannot have cliques of dimension 2, then $K_{e}^{2}(G)$ cannot have cliques neither of dimension 2 nor of dimension 1, while $K_{e}^{r}(G)=K_{e}^{r-1}(G)$ for $r \geq 3$.

\section{Summarizing:}

Corollary 3.6 $q(G) \geq q\left(K_{e}(G)\right)$.

It is to notice that Fact 3.5 implies that two different cliques in $K_{e}(G)$ may share a number of vertices equal to a binomial coefficient. Let us consider in $G$ two cliques of dimension $h$ and $k$, respectively, sharing $t$ vertices. These vertices induce a clique in $G$, hence in $K_{e}(G)$ the two cliques become two cliques of dimension $\left(\begin{array}{l}h \\ 2\end{array}\right)$ and $\left(\begin{array}{l}k \\ 2\end{array}\right)$, and they share $\left(\begin{array}{l}t \\ 2\end{array}\right)$ vertices.

The correlation between the chromatic number of a graph $G$ and the chromatic number of its edgeclique graph follows the same rule of the correlation between the corresponding cliques of these graphs.

Theorem 3.7 [1] The chromatic number of an edge-clique graph $K_{e}(G)$ has as upper bound the value $\left(\begin{array}{c}\chi(G) \\ 2\end{array}\right)$, where $\chi(G)$ is the chromatic number of $G$.
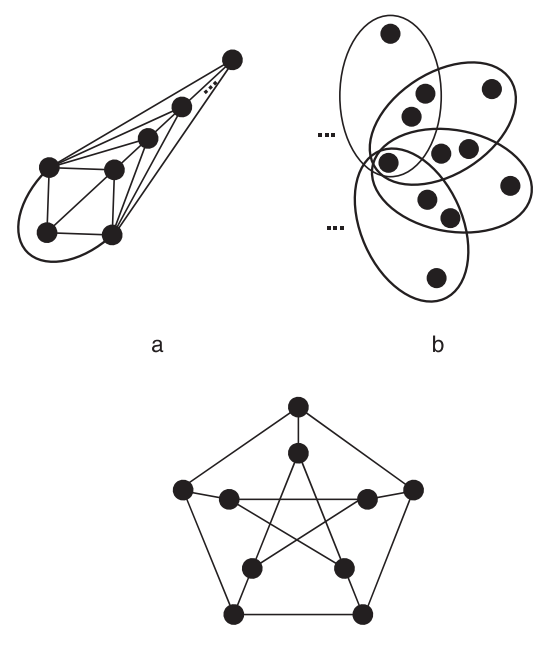

Figure 3: a. A planar graph $G$ with some cliques of dimension 4; b. its (not planar) edge-clique graph $K_{e}(G)$. For the sake of clearness, the edges of the cliques - represented by ovals - are not drawn; c. Petersen graph.

From Theorem 3.7 it is possible to derive that if in $G$ there are not cliques of dimension greater than 3:

- $\chi\left(K_{e}(G)\right) \leq \chi(G)$;

- $\chi\left(K_{e}(G)\right)<\chi(G)$ if $\chi(G)$ is even (see Fig. 4).

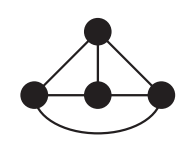

a

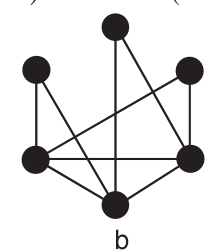

Figure 4: a. A graph $G$ with $\chi(G)=4$; b. Its $K_{e}(G)$ with $\chi\left(K_{e}(G)\right)=3$.

\section{Edge-clique graph of an outerplanar graph}

In this section we study the edge-clique graph of an outerplanar graph.

First, we recall a characterization of outerplanar graphs:

Theorem 4.1 [5] A graph is outerplanar if and only if it contains no subgraph homeomorphic to $K_{4}$ or $K_{2,3}$ by a homeomorphism that contracts degree-2 vertices.

As consequence of Theorem 4.1, a triangle is the biggest clique an outerplanar graph can contain as subgraph. From Theorem 3.4 and from the fact that an outerplanar graph is $K_{4}$ free, we deduce that its edge-clique graph is planar. Here we prove that it remains an outerplanar graph and we characterize its special structure. 
Lemma 4.2 Let $G$ be an outerplanar graph and let $K_{e}(G)$ be its edge-clique graph. Then, two triangles in $K_{e}$ can share at most one vertex (see Fig. 5.a).

Proof: Let us suppose by contradiction that two triangles sharing an edge exist in $K_{e}(G)$, then in $G$ the corresponding triangles would share a pair of edges, i.e. they would be the same triangle, against the initial hypothesis that $G$ is simple (see Fig. 5.b).

Lemma 4.3 Given an outerplanar graph $G$, its edge-clique graph $K_{e}(G)$ does not contain cycles longer than three.

Proof: Let us suppose, by contradiction, that a $k$ cycle, $k>3$, exists in $K_{e}(G)$. This implies that in $G$ there is a wheel of $k$ triangles, each one sharing an edge with its neighbors contradicting the fact that $G$ is outerplanar (see Fig.5.c).

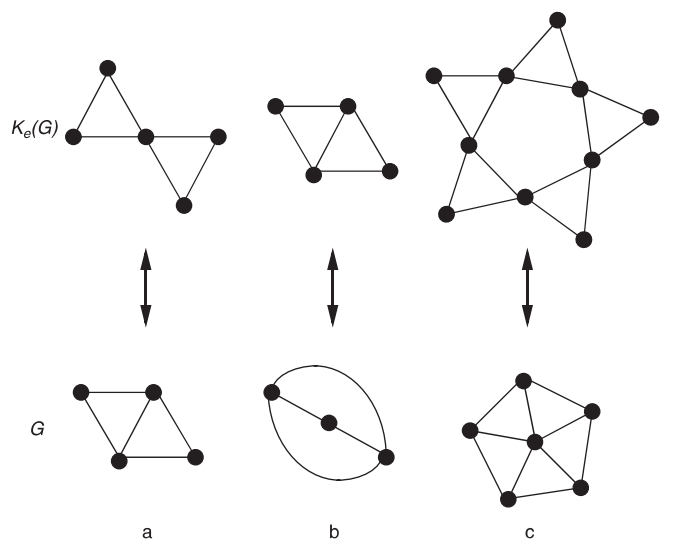

Figure 5: Some combinations of triangles: a. the only allowed combination in edge-clique graph of an outerplanar graph; b. and c. forbidden combinations in edge-clique graph of an outerplanar graph.

Definition 4.4 A tree of triangles is a simple graph in which:

- every edge is involved in exactly one triangle (three vertices and three edges);

- two triangles can share only one vertex and each vertex belongs to at most two triangles;

- the clique graph of a tree of triangles is a tree (see Fig. 6).

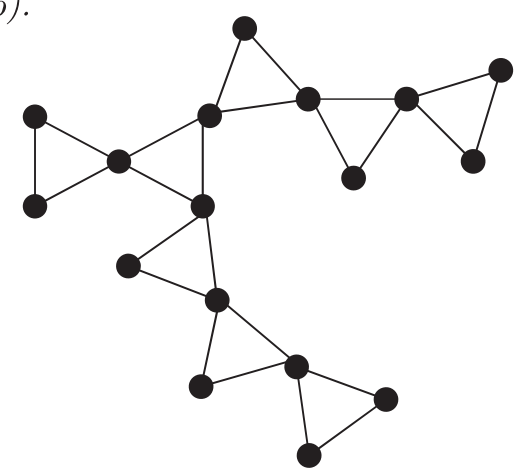

It is straightforward to see that:

Fact 4.5 A tree of triangles is an outerplanar graph and each vertex has degree either 2 or 4.

Theorem 4.6 Let $H=K_{e}(G)$. Then $G$ is an outerplanar graph $G$ if and only if $H$ is the disjoint union of isolated vertices, isolated triangles and trees of triangles.

Proof: In view of Fact 3.5, cliques of dimension 2 and 3 in $G$ become isolated vertices and triangles, respectively, in $K_{e}(G)$. Theorem 4.1 guarantees that cliques of dimension 4 cannot exist.

Lemma 4.2 and Lemma 4.3 specify that groups of connected triangles in $K_{e}(G)$ can only constitute trees of triangles.

The converse is straightforward.

Theorem 4.7 Given an outerplanar graph $G$, its edge-clique graph $K_{e}(G)$ is an outerplanar graph.

Proof: The statement descends from Theorem 4.6 and Fact 4.5 .

\section{The $\lambda$-coloring problem}

The radio frequency assignment problem consists in assigning radio frequencies to transmitters at different locations without causing interference. This situation can be modeled by a graph, whose vertices are the radio transmitters/receivers, and the adjacencies indicate possible communications and, hence, interference. On this model, the radio frequency assignment problem is then transformed into a coloring problem, where colors represent possible frequencies.

In the $\lambda$-coloring problem [16] 'close' transmitters must receive different frequencies and 'very close' transmitters must receive frequencies that are at least two apart. The aim is to minimize the number of used frequencies. In terms of graphs, two vertices are 'very close' if they are adjacent, while they are 'close' if lie at distance two in the graph.

More formally:

Definition 5.1 [3] $A \lambda$-coloring of a graph $G$ is a function $f$ from the vertex set $V(G)$ to the set of all nonnegative integers such that

1. $|f(x)-f(y)| \geq 2$ if $d(x, y)=1$ and

2. $|f(x)-f(y)| \geq 1$ if $d(x, y)=2$.

The $\lambda$-number of $G$ (simply $\lambda(G)$ or $\lambda$ for short, when no confusion arises) is the smallest number of colors necessary to $\lambda$-color $G$ minus one, i.e. the biggest used color in any optimal $\lambda$-coloring.

Figure 6: A tree of triangles. 
The $\lambda$-coloring problem has been very studied in the last decade, for its interesting applications in the field of mobile computing, so many results are present in the literature. Namely, for some classes of graphs - such as paths, cycles, tilings, wheels and $k$-partite graphs - tight bounds for the number of colors necessary for a $\lambda$-coloring are known and such a coloring can be computed efficiently. Nevertheless, in general both determining the minimum number of necessary colors [16] and determining if this number is $<k$ for every fixed $k \geq 4$ [14] is NPcomplete. Therefore, for many classes of graphs such as chordal graphs [24], interval graphs [12], unigraphs [8] - approximate bounds have been looked for.

In Table 1 most of the known results related to $\lambda$ coloring of specific classes of graphs are summarized.

In this paper we investigate on $\lambda$-coloring of edgeclique graphs. First, we can observe that a translation of Theorem 3.7 for $\lambda$ does not hold. Indeed, $\lambda(G)=\lambda\left(K_{e}(G)\right)$ when $G$ is a triangle, as it is the fixed point for the $K_{e}$ operator. On the contrary, $\lambda(G)<\lambda\left(K_{e}(G)\right)$ for any $G$ clique of dimension greater than 3. Finally, $\lambda(G)>\lambda\left(K_{e}(G)\right)$ whenever $G$ is a non trivial graph with no cliques (e.g. $G$ is a simple cycle). Hence, it seems not possible to derive general bounds on $\lambda$; on the contrary, we have to handle the $\lambda$-coloring problem separately on each class of graphs.

Looking at the table and considering Theorem 3.3 it is easy to assert that results concerning the $\lambda$ coloring problem on chordal and strongly chordal graphs hold for $K_{e}(G)$, too. From the other hand, the edge-clique graph of a clique is still a clique (see Fact 3.1) and for it we can compute an optimal $\lambda$ coloring. In the previous section we have proven that the edge-clique graph of an outerplanar graph is outerplanar; it follows that the results given in [7] hold. More in general, results on $\lambda$-coloring are extended to edge-clique graphs for all classes of graphs $\mathcal{C}$ such that if $G$ is in $\mathcal{C}$ so is its edge-clique graphs. Concerning planar graphs, the results on $\lambda$-coloring planar graphs can be applied to edge-clique graphs of planar graphs without cliques of dimension 4 (see Theorem 3.4). Nothing we are able to say about edge-clique graphs of planar graphs with cliques of dimension 4 (cf. Fig. 3).

The edge-clique graph of a path, of a cycle with more than 3 vertices, of a hexagonal or squared tiling, of a tree and of a bipartite graph is a set of isolated vertices (see Fact 3.2), and hence it makes no sense to study the $\lambda$-coloring of such a graph because one color is trivially sufficient.
The edge-clique graph of a triangular tiling is a subgraph of a triangular tiling (see Fig. 8), then for it we can consider the bound relative to triangular tiling, even if it is not tight. In fact, using techniques similar to those in [6], it is possible to show that $6 \leq \lambda \leq 7$ (see Fig. 8.b). It remains an open problem to understand which is the exact value of $\lambda$. Concluding, for almost all the classes for which bounds on $\lambda$ are known, it is possible to determine bounds on $\lambda$ for their edge-clique graph. In Section 6 we take a step forward on completing Table 1 providing a linear time algorithm computing a $\lambda$-coloring of the edge-clique graph of a threshold graph.

\section{$6 \lambda$-coloring the edge-clique graph of a threshold graph}

In this section we completely describe the edgeclique graph $K_{e}(G)$ of a threshold graph $G$, and then we study the $\lambda$-coloring problem on $K_{e}(G)$, providing a linear time algorithm that $\lambda$-colors $K_{e}(G)$.

Let us consider the edge-clique graph $K_{e}(G)=$ $\left(V^{\prime}, E^{\prime}\right)$ of a threshold graph $G=\left(V_{K} \cup V_{S}, E\right)$. As $G$ is defined and from Fact $3.5, K_{e}(G)$ contains a clique $K^{\prime}$ of dimension $\left(\begin{array}{c}\left|V_{K}\right| \\ 2\end{array}\right)$.

For what concerns the edges between $K$ and $S$, consider the general vertex $v_{i} \in V_{S}$ and its neighborhood $N\left(v_{i}\right)=u_{1}, u_{2}, \ldots u_{\operatorname{deg}\left(v_{i}\right)} \subseteq V_{K}$ (see Fig. 7.a). The subgraph induced in $G$ by $\left\{v_{i}\right\} \cup N\left(v_{i}\right)$ is a clique of dimension $\operatorname{deg}\left(v_{i}\right)+1$. Therefore, such a clique corresponds to a clique $K_{i}^{\prime}$ in $K_{e}(G)$ of dimension $\left(\begin{array}{c}\operatorname{deg}\left(v_{i}\right)+1 \\ 2\end{array}\right)$. This clique has $\operatorname{deg}\left(v_{i}\right)$ vertices outside $K^{\prime}$, while shares all its other vertices with $K^{\prime}$ (see Fig. 7.b).

The previous reasonings must be replicated for each vertex in $V_{S}$ (see Fig. 9). It follows that $K_{e}(G)$ is constituted by:

- a clique $K^{\prime}$ of dimension $\left(\begin{array}{c}\left|V_{K}\right| \\ 2\end{array}\right)$ (coming out from $K$ in $G$ );

- $\left|V_{S}\right|$ cliques $K_{i}^{\prime}$ of dimension $\left(\begin{array}{c}\operatorname{deg}\left(v_{i}\right)+1 \\ 2\end{array}\right), i=$ $1,2, \ldots,\left|V_{S}\right|$ (coming out from each $\left\{v_{i}\right\} \cup N\left(v_{i}\right)$ in $G)$.

Cerioli and Szwarcfiter [11] show that this is also a sufficient condition, i.e. if $K_{e}(G)$ has these properties, then $G$ is a threshold graph.

Observation 6.1 As a threshold graph is defined, it must be $\left(K_{1}^{\prime} \cap K^{\prime}\right) \supseteq\left(K_{2}^{\prime} \cap K^{\prime}\right) \supseteq \ldots \supseteq\left(K_{|S|}^{\prime} \cap K^{\prime}\right)$. 


\begin{tabular}{|c|c|c|c|}
\hline & Classes of Graphs & Bounds on $\lambda$ & Complexity \\
\hline$\sqrt{ }$ & Paths & $\lambda=2,3$ or $4[16]$ & $\mathrm{P}[16]$ \\
\hline$\sqrt{ }$ & Cycles & $\lambda=4[16]$ & $\mathrm{P}[16]$ \\
\hline$\sqrt{ }$ & Cliques & $\lambda=2 \Delta-2[16]$ & $\mathrm{P}[16]$ \\
\hline$\sqrt{ }$ & Hexagonal Tiling & $\lambda=5[2,6]$ & $\mathrm{P}[2,6]$ \\
\hline$\sqrt{ }$ & Squared Tiling & $\lambda=6[2,6]$ & $\mathrm{P}[2,6]$ \\
\hline & Triangular Tiling & $\lambda=8[2,6]$ & $\mathrm{P}[2,6]$ \\
\hline$\sqrt{ }$ & Trees & $\lambda=\Delta+1$ or $\Delta+2[16]$ & $\mathrm{P}[12]$ \\
\hline$\star$ & Threshold & $\lambda \leq 2 \Delta[8]$ & $\mathrm{P}[8]$ \\
\hline$\star$ & $\begin{array}{l}\text { Outerplanar: } \\
\qquad \begin{array}{l}\Delta \geq 8 \\
4 \leq \Delta \leq 7 \\
\Delta=3 \\
\Delta=3 \text { triangle free }\end{array}\end{array}$ & $\begin{array}{l}\lambda \leq \Delta+2 \\
\lambda \leq 11 \\
\lambda \leq \Delta+5 \\
\lambda \leq \Delta+4[7]\end{array}$ & \\
\hline$\sqrt{ }$ & Strongly Chordal & $\lambda \leq 2 \Delta[12]$ & \\
\hline & Unigraphs & $O(\Delta)[8]$ & \\
\hline$\sqrt{ }$ & Bipartite & $\lambda=\Theta\left(\Delta^{2}\right)[3]$ & NP-complete [3] \\
\hline & Planar & $\begin{array}{l}\lambda \leq 2 \Delta+25[17] \\
\lambda \leq \frac{5}{3} \Delta+90[20]\end{array}$ & $\begin{array}{l}\text { NP-complete } \\
{[3,15]}\end{array}$ \\
\hline$\sqrt{ }$ & Chordal & $\lambda \leq \frac{1}{4}(\Delta+3)^{2}[24]$ & NP-complete [3] \\
\hline & Split & $\lambda=\Theta\left(\Delta^{1.5}\right)[3]$ & NP-complete [3] \\
\hline
\end{tabular}

Table 1: Some known results about $\lambda$-coloring of specific classes of graphs. Symbol $\sqrt{ }$ at the beginning of a line indicates that the $\lambda$-coloring problem on edge-clique graphs of the corresponding graphs is solved; symbol $\star$ refers to results on edge-clique graphs achieved in the present paper.

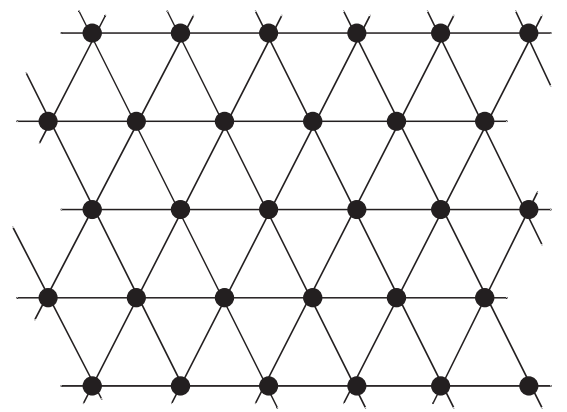

a

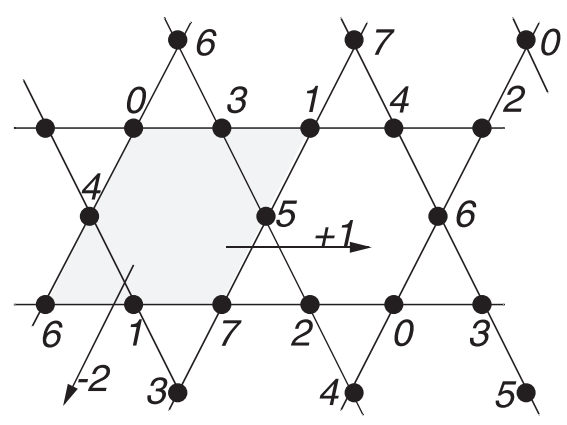

b

Figure 7: a. triangular tiling; b.its edge-clique graph. 


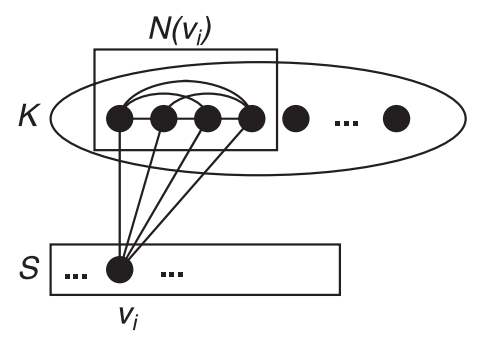

a

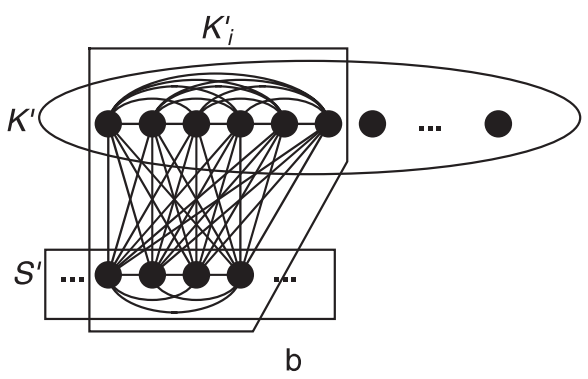

Figure 8: How $\left\{v_{i}\right\} \cup N\left(v_{i}\right)$ in $G$ becomes in $K_{e}(G)$.

Now we describe a new algorithm to $\lambda$-color an edge-clique graph $K_{e}(G)$ of a threshold graph $G$ by giving first an high level view and then all the details.

For our aim it is not restrictive to suppose that each vertex $v_{i} \in V_{S}$ has degree $\operatorname{deg}\left(v_{i}\right) \geq 2$. Indeed, if $\operatorname{deg}\left(v_{i}\right)=1$ for some $v_{i} \in V_{S}$, the unique edge $e_{i}$ incident to $v_{i}$ in $G$ produces a clique of dimension 1 , i.e. an isolated vertex, in $K_{e}(G)$. The $\lambda$-coloring of this vertex does not increase the value of $\lambda$. Under this assumption and from Observation 6.1 it follows that all vertices in $K_{S}^{\prime} \cap K^{\prime}$ are universal vertices, i.e. they are connected to all vertices in the graph; then, each vertex of $K_{e}(G)$ must receive a different color.

Let $S^{\prime}$ be $V^{\prime}-K^{\prime}$. As an edge-clique graph is defined, degree of each vertex in $K^{\prime}$ is never less than degree of any vertex in $S^{\prime}$. Hence, it is possible to sort all vertices of $K_{e}(G)$ according to their degree. From now on let us call $x_{1}, x_{2}, \ldots, x_{\left|K^{\prime}\right|}$ vertices in $K^{\prime}$ and $w_{1}, w_{2}, \ldots w_{\left|S^{\prime}\right|}$ vertices in $S^{\prime}$, so that the higher is their degree, the smaller is their index.

The algorithm labels vertices in $K_{e}(G)$ one by one, starting from vertices in $K^{\prime}$ and going on with vertices in $\left|S^{\prime}\right| . K^{\prime}$ is $\lambda$-colored with the first $\left|K^{\prime}\right|$ even colors [16], starting from the universal vertices to vertex $x_{\left|K^{\prime}\right|}$. The $\lambda$-coloring of vertices in $S^{\prime}$ works clique by clique, moving from vertices in $K_{\left|V_{S}\right|}^{\prime}$ to vertices in $K_{1}^{\prime}$, and requires a final adjustment phase in order to improve the $\lambda$-coloring.
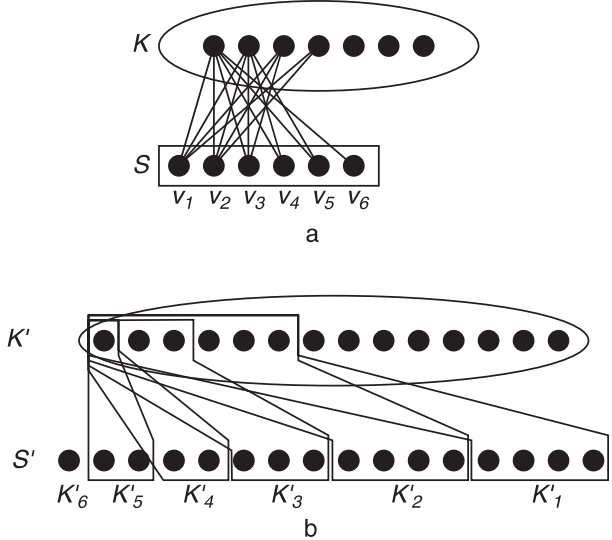

Figure 9: a. A threshold graph $G$; b. its edge-clique graph $K_{e}(G)$. For sake of clearness, the edges in the cliques are not drawn.

Namely, working on vertices in $K^{\prime}$, the not used (odd) colors are put in a queue. Let $c$ be a color in the queue. If either $c-1$ or $c+1$ are (even) colors assigned to a universal vertex, then $c$ is thrown out, because each vertex $w \in S^{\prime}$ is adjacent to each universal vertex and therefore $w$ cannot be labeled with c. Otherwise, $c$ is assigned to any vertex $w \in K_{r}^{\prime} \cap S^{\prime}$ such that neither $c-1$ nor $c+1$ are used to label some vertex in $K_{r}^{\prime} \cap K^{\prime}$. If this is not the case, $c$ is thrown out. After the queue is emptied, new colors must be used. Of course, if a color $c$ is assigned to a vertex $w \in K_{r}^{\prime} \cap S^{\prime}$, neither color $c-1$ nor $c+1$ can be used in the same clique $K_{r}^{\prime}$, then they are enqueued. After all vertices of $K_{e}(G)$ have been labeled, queue may be empty or may be not. If the queue contains some colors, the adjustment phase must be run, in order to decrease the number of used colors. In particular, the algorithm visits all vertices in $S^{\prime}$, and looks for vertices whose colors can be replaced so that colors in queue are used and the biggest colors are thrown out.

Now, we trace the details of the previous algorithm.

ALGORITHM $\lambda$-COL- $K_{e}$-THRESHOLD

Input: Edge-clique graph $K_{e}(G)=\left(V^{\prime}, E^{\prime}\right)$ of a threshold graph $G=(K \cup S, E)$.

Output: A $\lambda$-coloring for $K_{e}(G)$.

Step 1. $\lambda$-Color $K^{\prime}$

for $i=1$ to $|K|$ do

label $x_{i}$ with (even) color $2 i-2$;

if $\left(x_{i}\right.$ is not universal)

then

enqueue (odd) color $2 i-1$; 
Step 2. $\lambda$-Color $S^{\prime}$

for $i=\left|V_{S}\right|$ downto 1 do

new $\leftarrow$ FALSE;

while ( $\exists$ a vertex $w \in K_{i}^{\prime} \cap S^{\prime}$ not labeled yet) do

if (queue-is-not-empty) and (new=FALSE)

then

dequeue color $c$;

if ( $c$ is feasible to $\lambda$-color $w$ )

then label $w$ with color $c$

else throw color $c$ out;

else

consider the first new color $c$;

if ( $c$ is feasible to $\lambda$-color $w$ )

then label $w$ with color $c$

else enqueue color $c$;

new $\leftarrow$ TRUE;

Step 3. Adjustment phase (to be run only if

queue is not empty)

while (queue-is-not-empty)do

consider vertex $w_{\text {cmax }} \in S^{\prime}$ having maxi-

mum color $c_{\max }$;

dequeue $c$;

if $\left(\exists w \in S^{\prime}\right.$ having color $c_{w}$ s.t. labeling $w$ with $c$ and $w_{c \max }$ with $c_{w}$ provides a feasible $\lambda$-coloring)

then

relabel $w_{\text {cmax }}$ with color $c_{w}$;

relabel $w$ with color $c$;

if color $c_{\max }-1$ is in queue then throw it out.

Before presenting the theorem that prove the correctness and complexity of the previous algorithm, we want to better clarify Step 2. Namely, boolean variable new is necessary for correctly labeling one at a time vertices in clique $K_{i}^{\prime}$ : when queue is empty, a new color $c$ must be considered and, if it is feasible, new guarantees that colors $c+2, c+4, \ldots$ are used to label next vertices in the same clique $K_{i}^{\prime}$; nevertheless, when the algorithm moves to clique $K_{i+1}^{\prime}$, new switches its values so that colors $c+1, c+3, \ldots$ are extracted by queue.

Theorem 6.2 Algorithm $\lambda-C O L-K_{e}-$ THRESHOLD correctly computes a $\lambda$-coloring of the edge-clique graph $K_{e}(G)$ of a threshold graph $G$ in $O\left(\left|V^{\prime}\right|\right)$ time using no more than $\left|V^{\prime}\right|+\left|K_{|S|}^{\prime} \cap K^{\prime}\right|+\left|K_{1}^{\prime} \cap K^{\prime}\right|$ colors.

Proof: As the algorithm is designed, the produced coloring is a feasible $\lambda$-coloring.

In order to show that the algorithm uses no more than $\left|V^{\prime}\right|+\left|K_{|S|}^{\prime} \cap K^{\prime}\right|+\left|K_{1}^{\prime} \cap K^{\prime}\right|$ colors, notice that - as all vertices in $K_{|S|}^{\prime} \cap K^{\prime}$ are universal no color can be reused, hence algorithm $\lambda$-COL- $K_{e^{-}}$
THRESHOLD uses at least $\left|V^{\prime}\right|$ colors. Furthermore, the algorithm throws out all (odd) colors close to colors used to label the universal vertices, i.e. $\left|K_{|S|}^{\prime} \cap K^{\prime}\right|$ colors. Finally, if the current vertex $w$ cannot be labeled with the dequeued color $c$, we observe that no other unlabeled vertex $w^{\prime}$ can, in view of Obs. 6.1, and therefore we throw $c$ out. In such a case, if $w \in K_{i}^{\prime}$ for some $i, c$ must be close to a color used to label a node $v \in K_{i}^{\prime} \cap K^{\prime}$. It follows that globally - no more than $\max _{i}\left|K_{i}^{\prime} \cap K^{\prime}\right|=\left|K_{1}^{\prime} \cap K^{\prime}\right|$ colors can be thrown out. Up to now, no more than $\alpha=\left|V^{\prime}\right|+\left|K_{|S|}^{\prime} \cap K^{\prime}\right|+\left|K_{1}^{\prime} \cap K^{\prime}\right|$ colors are used. To conclude the proof, we have to show that algorithm $\lambda$-COL- $K_{e}$-THRESHOLD uses no more than $\alpha$ colors, i.e. that Step 3 always empties queue. If the number $|S|$ of cliques $K_{i}^{\prime}$ is $|S|>1$, then Step 3 always runs successfully, since the colors close to enqueued colors have been assigned by Step 2 to vertices in the biggest $K_{i}^{\prime}$ s (i.e. smallest values of $i)$; therefore, it is always possible to find vertices $w$ in the smaller $K_{i}^{\prime}$ s willing to swap their colors. The worst case happens when $|S|=1$ and the unique resulting clique $K_{1}^{\prime}$ is as bigger as possible. In this case, $\left|K^{\prime}\right|$ and $\left|K_{1}^{\prime}\right|$ are both equal to $\left(\begin{array}{c}|K| \\ 2\end{array}\right)$, if $\operatorname{deg}\left(v_{1}\right)=|K|-1$, that is the maximum possible.

Observe that $K_{1}^{\prime}$ can be partitioned into $K_{1}^{\prime} \cap K^{\prime}$ and $K_{1}^{\prime} \cap S^{\prime}=S^{\prime}$. As the algorithm works, exactly $\left|K_{1}^{\prime} \cap K^{\prime}\right|$ colors are thrown out, and in queue there are $\left|K^{\prime}\right|-1-\left|K_{1}^{\prime} \cap K^{\prime}\right|$. A problem arises if $\left|K_{1}^{\prime} \cap S^{\prime}\right|$ is much bigger than the number of enqueued colors. Indeed, in this case, the algorithm would use many odd colors and would enqueue the corresponding even colors, but these enqueued colors could not be used in any way. As edge-clique graph of a threshold graph is defined, in this case, $\left|K_{1}^{\prime} \cap S^{\prime}\right|$ is exactly equal to $\left|K^{\prime}-K_{1}^{\prime}\right|$, and then all vertices in $S^{\prime}$ can be labeled with enqueued colors, but one; this last vertex uses a new consecutive color and hence Step 3 does not need to be executed because queue is empty.

To prove that the time complexity is linear in the number of vertices of $K_{e}(G)$, let us do the following considerations. Step 1 and 2 take time proportional to $\left|K^{\prime}\right|$ and $\left|S^{\prime}\right|$, respectively, hence $O\left(\left|V^{\prime}\right|\right)$ time, globally. Also Step 3 can be carried on in linear time, if the data structures are implemented in appropriate way, e.g. with a set of links connecting each used color $c$ with the vertex labeled $c$ and with the position (in queue or on vertices of $S^{\prime}$ ) of $c-1$ and $c+1$. In this way, it is sufficient to visit at most once all vertices in $S^{\prime}$, starting from vertices labeled with smaller colors, and this is equivalent to visit them following their numbering in decreasing fashion (from $w_{\left|S^{\prime}\right|}$ to $w_{1}$ ). 
Before concluding with an example showing how algorithm $\lambda$-COL- $K_{e}$-THRESHOLD works, we want to highlight that, for the structure of $K_{e}(G)$, it always hold: $\lambda\left(K_{e}(G)\right) \geq\left|V^{\prime}\right|+\left|K_{|S|}^{\prime} \cap K^{\prime}\right|-1$. This observation implies that, if the else statement in Step 2 is never executed, the algorithm finds the optimal solution; in any case, it always guarantees a number of colors linear in the degree of $K_{e}(G)$.

\subsection{An example}

Let $G=\left(V_{K} \cap V_{S}, E\right)$ be a threshold graph with $\left|V_{K}\right|=5$ and $\operatorname{deg}\left(v_{1}\right)=\operatorname{deg}\left(v_{2}\right)=4$ and $\operatorname{deg}\left(v_{3}\right)=2$ and with the resulting edge-clique graph depicted in Fig. 10.a. Step 1 of the algorithm labels all vertices in $K^{\prime}$ with consecutive even colors, throw color 1 out and enqueue all other odd colors from 3 to $2\left|K^{\prime}\right|-1$ (see Fig. 10.a and Fig. 10.b).

Step 2 labels vertices in $S^{\prime}$ starting from $K_{3}^{\prime}$ : it dequeues colors 3 and 5 and assigns them to vertices in $K_{3}^{\prime} \cap S^{\prime}$ (see Fig. 10.c).

When $K_{2}^{\prime}$ is considered, enqueued color 7 is not feasible; the same holds for colors 9 and 11; therefore they are thrown out (see Fig. 10.d). Vertices in $K_{2}^{\prime} \cap S^{\prime}$ are labeled with the next four colors in queue and queue is empty (see Fig. 10.e).

New colors are used to $\lambda$-color vertices in $K_{1}^{\prime} \cap S^{\prime}$, and consequently three close new colors are enqueued (see Fig. 10. e and 10.f).
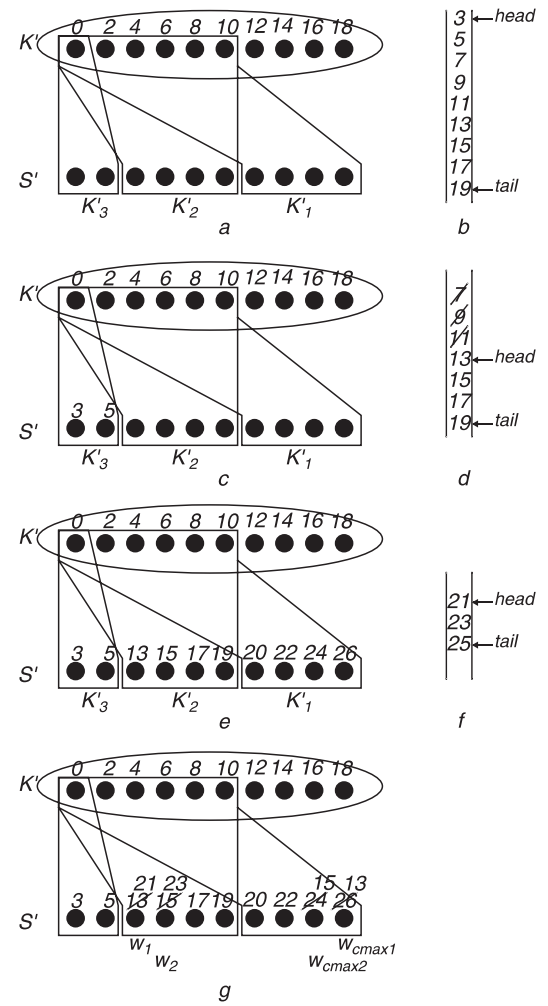

Figure 10: An example showing how algorithm $\lambda$ COL- $K_{e}$-THRESHOLD works.
Since queue is not empty, Step 3 must be run: vertex labeled with 26 is selected as $w_{\text {cmax }}$ and vertex labeled with 13 is selected as $w$; so $w_{\text {cmax }}$ is relabeled with 13 and $w$ with the first color in queue, i.e. 21; color 25 is dequeued. Since queue is not empty, this step is executed again, as shown in Fig. 10.e. At the end of Step 3 maximum used color is decreased from 26 to 23 .

\section{References}

[1] M.O. Albertson and K.L. Collins: Duality and Perfection for Edges in Cliques. J. of Combinatorial Theory., B/36:298-309, 1984.

[2] A.A. Bertossi, C.M. Pinotti and R.B. Tan: Efficient Use of Radio Spectrum in Wireless Networks with Channel separation between Close Stations. In Proceedings of 4th ACM Int. Workshop on Discrete Algorithms and Methods for Mobile Computing and Communications (DIAL M), 2000.

[3] H.L. Bodlaender, T. Kloks, R.B. Tan and J. van Leeuwen: $\lambda$-Coloring of Graphs. In Proceedings of STACS 2000, pages 395-406. Lectures Notes in Computer Science 1770, 2000.

[4] C.F. Bornstein and J.L. Szwarcfiter: Clique Graphs of Chordal and Path graphs. SIAM J. Disc. Math., 9: 331-336, 1996.

[5] A.Brandstadt,V.B.Le and J.P.Spinrad: Graph Classes: A Survey. SIAM Monographs on Discrete Mathematics and Applications, Philadelphia, 1999.

[6] T. Calamoneri and R. Petreschi: $\lambda$-Coloring of Regular Tiling. In Proceedings of First Cologne-Twente Workshop (CTW). Electronic Notes in Discrete Mathematics , 8, 2001. http://www.elsevier.nl/locate/endmCTW2001.

[7] T. Calamoneri and R. Petreschi: $L(2,1)-$ Labeling of Planar Graphs (Extended Abstract). In Proceedings of 5th ACM Int. Workshop on Discrete Algorithms and Methods for Mobile Computing and Communications (DIAL M), 2001, pages 28-33.

[8] T. Calamoneri and R. Petreschi: $\lambda$-Coloring Unigraphs. In Proceedings of LATIN 2002. Lectures Notes in Computer Science, 2002 (to appear).

[9] M.R.Cerioli: Grafos Clique de Arestas. Tech. Rep. ES438/97, Dept. of Engineering and Computer Systems, Fed. Univ. of Rio de Janeiro, 1997. 
[10] M.R.Cerioli and J.L. Szwarcfiter: A Characterization of Edge-Clique Graphs. Ars Combinatoria, 60: 287-292, 2001.

[11] M.R.Cerioli and J.L. Szwarcfiter: Edge Clique Graphs and Some Classes of Chordal Graphs. Discrete Mathematics, 242: 31-39, 2002.

[12] G.J. Chang and D. Kuo: The L(2,1)-labeling Problem on Graphs. SIAM J. Disc. Math., 9: 309-316, 1996.

[13] G. Chartrand, S.F. Kapoor,T.A.McKee and F. Saba: Edge clique Graphs. Graphs and Combinatorics, 7: 253-264, 1991.

[14] J. Fiala, T. Kloks and J. Kratochvíl: Fixedparameter Complexity of $\lambda$-Labelings. In Proceedings of Graph-Theoretic Concepts of Compu. Sci. (WG99), Lectures Notes in Computer Science 1665, pages 350-363, 1999.

[15] D.A. Fotakis, S.E. Nikoletseas, V.G. Papadoulou and P.G. Spirakis: NP-completeness Results and Efficient Approximations for Radiocoloring in Planar Graphs. In Proceedings of 25th Int.l Symp. on Math. Foundations of Compu. Sci. (MFCS 2000), 2000.

[16] J.R. Griggs and R.K. Yeh: Labeling graphs with a Condition at Distance 2. SIAM J. Disc. Math, 5: 586-595, 1992.

[17] J. van den Heuvel and S. McGuinnes: Colouring the square of a planar graphs. Report LSECDAM-99-06, Centre for Discrete and Applicable Mathematics, London School of Economics, London, U.K., 1999.

[18] T.R. Jensen and B. Toft: Graph Coloring Problems. John Wiley \& Sons, New York, 1995.

[19] L.T.Kuo,L.J. Stockmeyer and C.K. Wong: Covering Edges by Cliques with regard to keywork conflicts and intersection graphs. Communication of the ACM., 21: 135-139, 1978.

[20] M.Molloy and M.R. Salavatipour. Frequency channel assignment on planar networks. Manuscript, 2001.

[21] E. Prisner: Line graphs and generalizations - a survey. Congressus Numerantium, 67: 197-204, 1996.

[22] E. Prisner and J.L. Szwarcfiter: Recognizing Clique Graphs of Directed and Rooted Path graphs. Discr. Appl. Math., 94: 321-328, 1999.

[23] N. Robertson and J.H. Spencer: A Characterization of Clique Graphs. J. of Combinatorial Theory, B/10: 102-108, 1971.
[24] D. Sakai: Labeling Chordal Graphs: Distance Two Condition. SIAM J. Disc. Math, 7: 133140, 1994.

[25] A. Raychaudhuri: Intersection Number and Edge-Clique Graphs of chordal and strongly chordal graphs. Congressus Numerantium, 67: 197-204, 1988.

[26] A. Raychaudhuri: Edge-Clique Graphs of some important classes of graphs. Ars Combinatorica , 32: 269-278, 1991. 